\title{
N 68-32187
}

Differential Ion Pumping for Vacuum Deposition*

W. F. Leonard

Institute of Technology

Southern Methodist University

Dallas, Texas

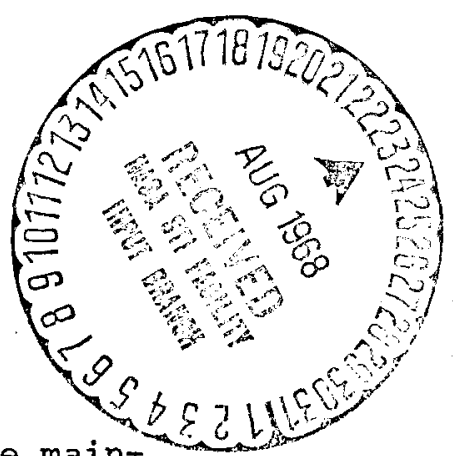

It is desirable to reduce the ultimate pressure that can be main-

tained during vacuum deposition to obtain films with low impurity content.

The major contributions to the total pressure during vacuum deposition

are outgasing from interior surfaces, leaks, permeation through gaskets,

back-streaming from the pump and the release of occluded gases from the

deposition material and holder. In a bakeable ultra high vacuum (UHV)

ion pump system evolution from all sources, except from the deposition

material and its holder, may be reduced to a low level. The pressure in

the substrate region may be reduced by reducing the conductance between

the source and substrate regions. In our preparation of thin films for

transport studies, the concept of differential pumping ${ }^{(1)}$ has been utilized

in a bakeable UHV ion pump system to achieve lower pressures in the vicinity

of the substrate.

The differentially pumped evaporator is shown in figure 1. The basic system consists of two bakeable chambers - a substrate chamber and a source chamber - connected by a plate with a small conductance tube which allows the vapor to traverse to the substrate. This arrangement confines the gases generated by the source to the source chamber during evaporation and maintains a large differential pressure. Thus, deposition can be made at lower pressures because only a small portion of the source gas load exists in the substrate chamber. Both chambers are ejectronicaliy pumped il 40 Iiter/sec Vac Ion pumps) and have ultimate pressures of $5 \times 10^{-11}$ Torr. Each chamber *Work supported by NASA Grant NGR 44-007-006.

(1) Schuetze, H. J. and Henning, K. E., Res./Dev., 64 (May 1965). (FST)
PAgEs C
0,15
H.C. 3.20
Cate
$M \cdot F . \$_{M}, S$ 
was assembled from $15.24 \mathrm{~cm}$ stainless steel fittings which were modified by the addition of $3.81 \mathrm{~cm}$ ports to accommodate sources, substrates, crystal oscillators, etc. All flanges are sealed by the use of copper gaskets, and heater strips are attached directly to the chamber walls to provide bakeout temperatures up to $400^{\circ} \mathrm{C}$.

The deposition source is a $2 \mathrm{KW}$ self accelerated electron beam gun and is located $2.54 \mathrm{~cm}$ below the conductance tube and $28 \mathrm{~cm}$ from the substrate. The conductance tube used has the dimensions shown in the insert of figure 2. The calculated conductance for this configuration is 0.46 liters/sec for air.

Pressure variations in the substrate chamber were measured with a Varian partial pressure ionization gauge (PPG) which has a mass range of 1-70AMU and in the source chamber a Bayard-Alpert type ionization gauge was used.

The characteristics of the conductance tube were determined by substituting a variable leak valve as a gas load in place of the electron beam gun. With a nitrogen leak the substrate chamber remained in the $10^{-10}-10^{-9}$ Torr region for pressures ranging up to $2.5 \times 10^{-6}$ Torx in the source chamber. Figure 2 is a plot of the actual performance of the conductance tube with the nitrogen leak. The PPG was taken as the pressure reference and was used to calibrate the Bayard-Alpert gauge. During actual deposition with copper as a source material, the pressure in the substrate chamber remained below $7.5 \times 10^{-8}$ Torr for deposition rates between $10-20 \stackrel{\circ}{\mathrm{A}} / \mathrm{sec}$. Attempts to increase the deposition rate have been hindered because the conductance tube, which is at ground potential, perturbs the electric field of the electron beam gun at large powers. 
Partial pressures were also measured before and during deposition of copper with the PPG system. The predominate peaks in both cases were $\mathrm{H}_{2}$ ' $\mathrm{H}_{2} \mathrm{O}$, and $\mathrm{N}_{2}$ with traces of $\mathrm{CO}_{2}$. During deposition, a proportionally larger increase of $\mathrm{H}_{2}$ and $\mathrm{CO}_{2}$ from the source material and gun occured.

Various techniques not yet incorporated in the system exist to further reduce the gases in the substrate chamber during deposition. For example, a shutter may be placed at the aperature of the conductance tube to allow initial degasing of the source material without affecting the pressure in the substrate chamber. Also, since water vapor is a predominant peak, the conductance tube could be cooled to liquid nitrogen temperature in order to cut down the conductance for water vapor. Even without these improvements the system performance is attractive since differential pressures of $200 / 1$ or better were easily obtained under heavy gas loads in the source chamber. 
Figure 1. Differentially pumped vacuum system for thin film evaporation: A - substrate chamber and B - source chamber.

Figure 2. Variation of pressure in substrate chamber with a variable nitrogen leak in source chamber. The conductance tube is shown in the insert $\left(D_{1}=3.18 \mathrm{~mm}, D_{2}=6.35 \mathrm{~mm}\right.$, and $\left.\mathrm{L}=25.4 \mathrm{~mm}\right)$. 


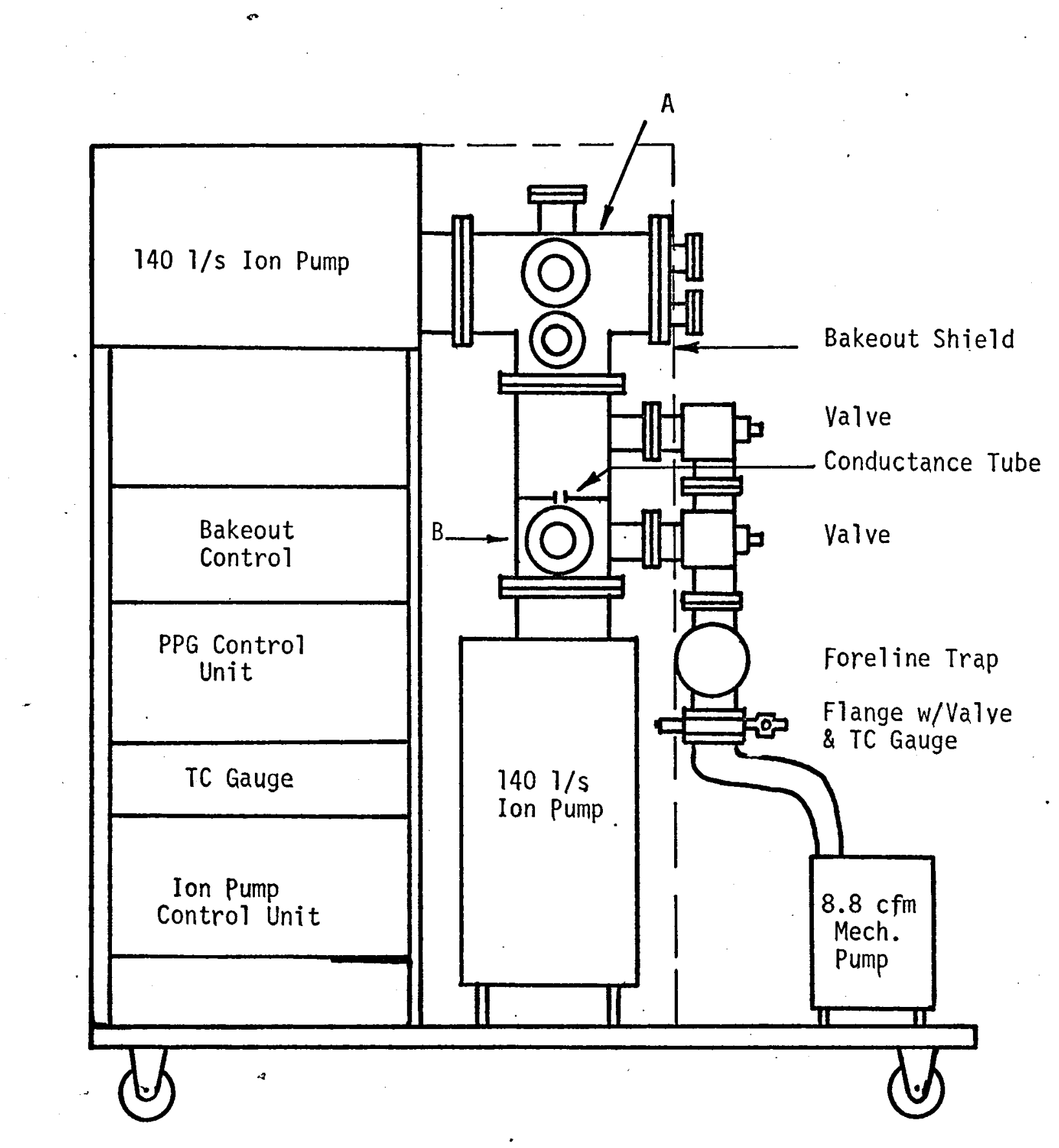

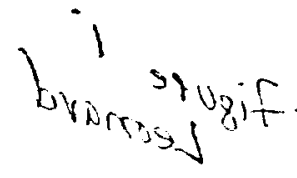




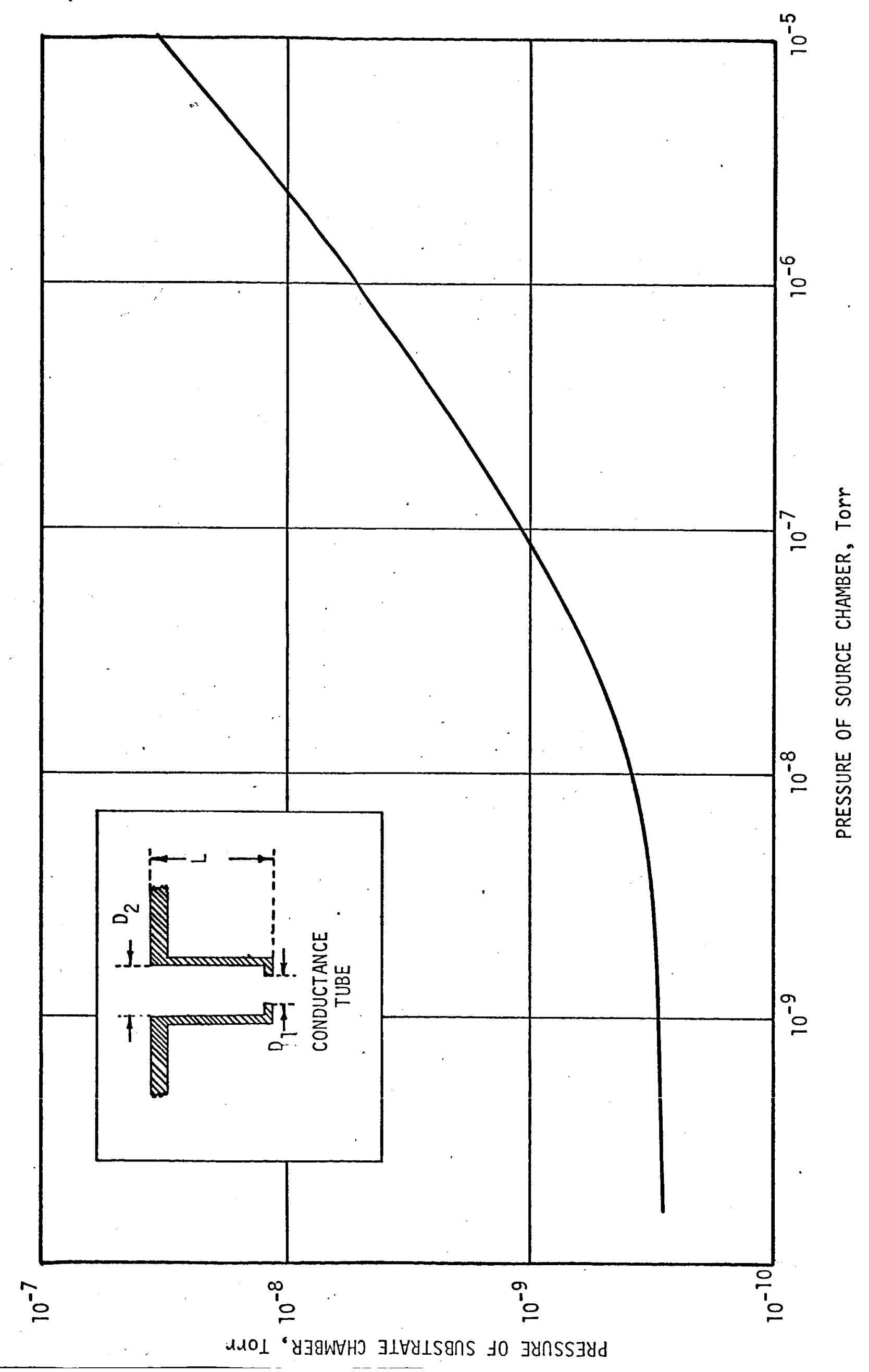

